\section{La inhibición a participar y su nexo con percibir una tutoría diferente}

\author{
MAYRA GARI, JEHU IPUTO
}

\section{How do students who feel "not free to participate" perceived their tutorial sessions in problem based learning?}

Background: Verbal interactions are paramount to develop cognitive and social skills of students taught using problem based learning in small group tutorials. Responses to a questionnaire showed that $27 \%$ of second year medical students did not feel free to participate in their groups Aim: To explore if these students may have different perceptions respect to their peers, regarding the functioning of their tutorials. Material and Methods: The answer to the item "feel free to participate" was the criterion to allocate students in two groups, study $(n=25)$ and control $(n=66)$. The means of the perceptions were compared between groups in the 15 items' questionnaire. Results: Students in the study group had different perceptions in eight of the items. These students enjoyed and considered interesting the topics addressed in the tutorials less frequently. Also they did not study all learning issues, did not always understand the case and foresaw less opportunities to participate in their groups. Conclusions: Quiet students perceived tutorials different than their more active peers. Those differences concerned motivation and a mixture of cognitive (individual) and social (rest of the group) strategies that were not conducive to build a suitable learning environment for those students in their respective tutorial groups.

(Rev Med Chile 2015; 143: 1191-1197)

Key words: Education, medical; Collaborative learning; Problem based learning.
Facultad de Ciencias de la Salud.

Universidad Walter Sisulu.

Mthatha. África del Sur.

Recibido el 25 de febrero de

2015, aceptado el 30 de junio de 2015.

Correspondencia a:

Dra. Mayra Gari

Walter Sisulu University. Private

Bag X1. Mthatha.

Eastern Cape. South Africa

mgari@wsu.ac.za

mgc1114@yahoo.com
I

a Facultad de Medicina de la Universidad Walter Sisulu (UWS) abrió sus puertas a los primeros doce estudiantes en el año 1985 en la entonces denominada Universidad de Transkei. La visión de la facultad siempre ha sido la formación de recursos humanos capaces de transformar responsablemente el panorama sanitario del país, y su política de admisión ofrece oportunidades para que estudiantes de áreas rurales se formen como médicos, muchos de los cuales retornan a prestar sus servicios a la provincia ${ }^{1}$. Los pilares pedagógicos que sostienen el diseño curricular del Grado de Medicina de esta universidad son la educación en y para la comunidad y el aprendizaje basado en la solución de problemas $(\mathrm{ABP})^{2}$.

La política de admisión de la UWS pondera de modo similar los resultados académicos de la enseñanza precedente así como las cualidades socio-culturales del candidato ${ }^{2}$. Tres cuartas partes de los alumnos de nuevo ingreso vienen directamente de la enseñanza media y el resto proviene de carreras de ciencias o ciencias de la salud.

El currículo está organizado en tres fases y cuatro temas, de manera que las asignaturas del ciclo básico biomédico preceden a las del ciclo clínico, pero con las correspondientes integraciones horizontal y vertical favorecidas por el método de instrucción seleccionado: ABP. La primera fase, dedicada al aprendizaje de la estructura y función normales del organismo humano, consta de seis bloques agrupados sistémicamente. La duración promedio de dichos bloques es de 8 semanas y los 
estudiantes son introducidos a un nuevo caso o problema semanalmente. El diseño pedagógico de los problemas aplica el paradigma bio-psico-social del ser humano, lo que diversifica las necesidades de aprendizaje que emanan de cada caso. La secuencia de los problemas ofrece opciones para que la transferencia de conocimiento intra e inter bloque pueda ser ejercitada ${ }^{3}$.

Los beneficios cognitivos del ABP como método de instrucción ${ }^{4}$ dependen de la calidad de las interacciones verbales que tienen lugar en los grupos de tutoría ${ }^{5}$, al tiempo que dichas interacciones, de ser socialmente favorables, van estimulando la evolución del grupo hacia un equipo $^{6}$. Este enfoque socio-cognitivo del aprendizaje se enriquece y complejiza si le adicionamos su dimensión cultural. Los valores y conductas que forman parte de cada cultura, se manifiestan en las interacciones que tienen lugar en los grupos, y por lo tanto influyen en el funcionamiento grupal y la calidad de su dinámica ${ }^{7}$. Es deseable que en el transcurso de estas interacciones el estudiante despliegue compromiso y responsabilidad con su aprendizaje y con el del grupo, lo que se manifiesta en cómo ocurren las regulaciones, individual (auto- y co-) y grupal, en aras de desarrollar una dinámica eficiente que coexista con un aprendizaje de calidad para todos los integrantes del grupo ${ }^{8}$.

En la UWS, en cada bloque, se crean nuevos grupos de tutoría y los integrantes (10-12) son asignados al azar, pero respetando la representación proporcional académica y cultural. Un estudio sobre el funcionamiento de las tutorías en el segundo año de la carrera de medicina en esta universidad reveló que $27 \%$ de los estudiantes encuestados sintió inhibiciones a participar en las interacciones verbales de sus grupos ${ }^{9}$. Este hallazgo nos motivó a explorar si estos individuos tienen diferentes percepciones sobre sus tutorías, en comparación a sus compañeros sin inhibiciones a participar.

\section{Material y Método}

El presente estudio pertenece a la categoría de investigación cuantitativa y tiene continuidad con un trabajo que exploró la percepción de los estudiantes sobre los factores que ellos consideraron influyen en el funcionamiento de sus tutorías ${ }^{9}$. Dos grupos focales de 10 estudiantes cada uno, decidieron los ítems y, en conjunto, participaron en la posterior elaboración y validación del contenido de la encuesta ${ }^{9}$. El análisis factorial con extracción de mínimo rango se realizó con el programa FACTOR, y se empleó el alfa ordinal como indicador de la fiabilidad del instrumento. Los ítems se agruparon en dimensiones social, motivacional, cognitiva y del estudio auto-regulado de las tutorías.

La afirmación "me sentí libre de participar en la pizarra o alrededor de la mesa" fue uno de los ítems que formó parte de dicha encuesta, y la asignación numérica de sus respuestas, desde el cero (no ocurrió en ningún bloque) hasta el cuatro (ocurrió en todos los bloques), vinculó la presencia del ítem al número de bloques. Veinticinco estudiantes $(27,4 \%)$ declararon sentirse inhibidos a participar en las sesiones de tutorías en dos o más bloques, mientras que $72,6 \%$ de ellos expresó tener inhibiciones en un bloque (28 estudiantes), o en ninguno (38 estudiantes). Estos rangos de respuestas a la afirmación anteriormente expresada fueron el criterio de asignación de los estudiantes a un grupo estudio $(\mathrm{n}=25)$ y al grupo control $(n=66)$.

En ambos grupos, estudio (GE) y control (GC), las respuestas a cada ítem se organizaron de acuerdo al rango numérico en el que fueron respondidas $(0,1,2,3$ y 4$)$ y se cuantificaron según categoría. A cada ítem se le calculó su media y desviación estándar. Las medias (M) se compararon ( $\mathrm{t}$-test) para conocer si las diferencias entre los grupos no fueron atribuibles a la casualidad, aceptando la significación estadística con un margen de error hasta de 5\%. La estadística fue calculada con el programa Epi Info 6.

\section{Operacionalización de variables}

GE: estudiantes que escogieron 0 ó 1 ó 2 a la aseveración "me sentí libre de participar en la pizarra o alrededor de la mesa"

GC: estudiantes que respondieron con los números 3 ó 4 a dicha afirmación.

\section{Resultados}

En ocho ítems las percepciones de los estudiantes del GE fueron diferentes $(\mathrm{p}<0,05)$ a las percepciones del GC (Tabla 1). Los 25 estudiantes que confesaron sentir inhibiciones a interactuar 


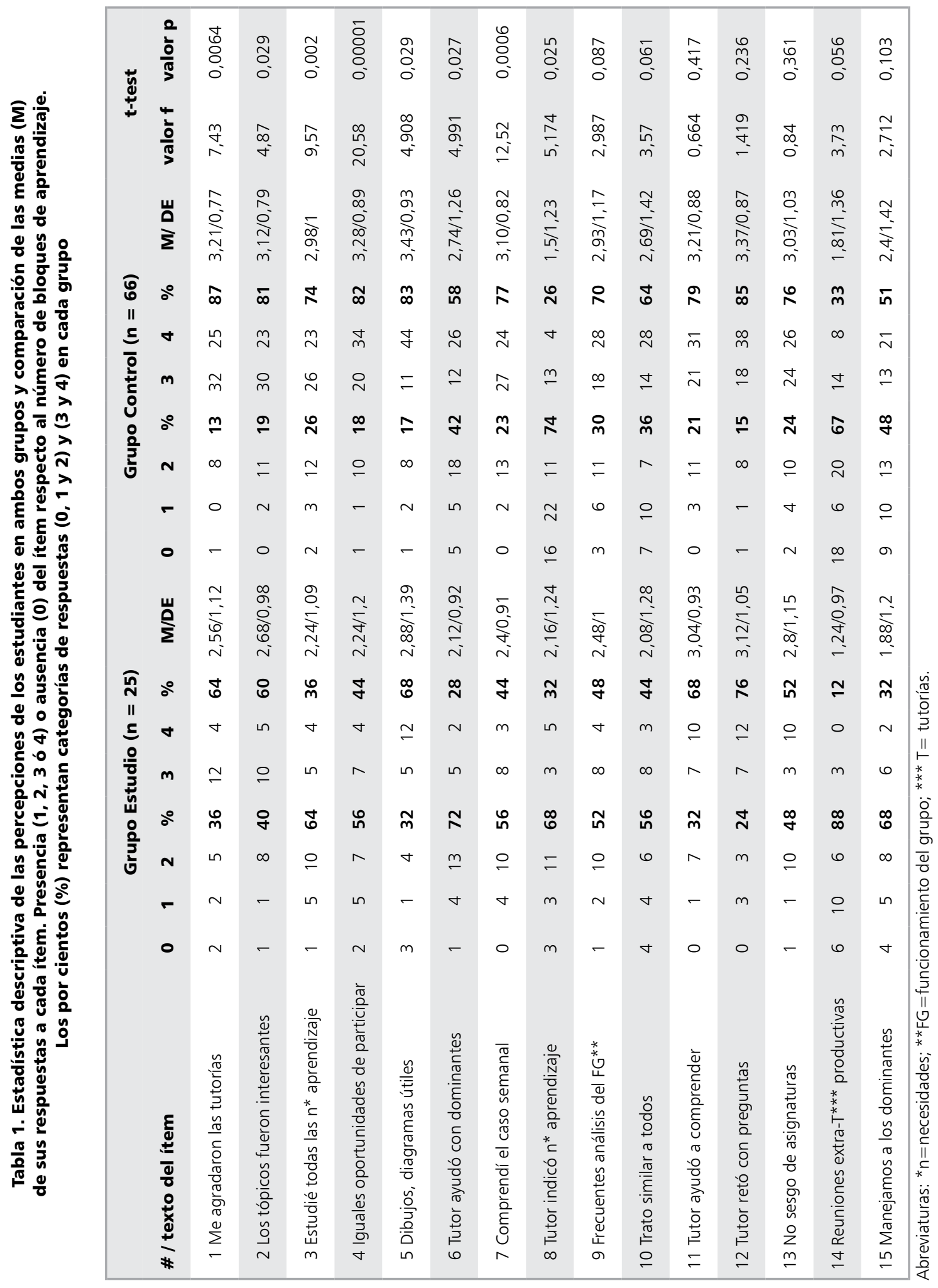


verbalmente en las tutorías también declararon menor agrado por las mismas (ítem $1 \mathrm{M}=2,56 \mathrm{vs}$ $3,21)$, los tópicos tratados fueron menos interesantes (ítem $2 \mathrm{M}=2,68$ vs 3,12), y comprendieron un menor número de casos semanales (ítem $7 \mathrm{M}=2,4$ vs 3,09$)$.Con menor frecuencia estudiaron todos los tópicos que fueron necesidades de aprendizaje(ítem $3 \mathrm{M}=2,24$ vs 2,98), no tuvieron iguales oportunidades de participar (ítem $4 \mathrm{M}=2,24$ vs 3,30 ), y se beneficiaron menos de los dibujos y diagramas para aprender (ítem $5 \mathrm{M}=2,88$ vs 3,44 ). También percibieron que su tutor les ayudó con menos frecuencia con el manejo de los estudiantes dominantes del grupo (ítem $6 \mathrm{M}=2,12$ vs 2,78 ) y con más frecuencia les dijo los tópicos a considerar como necesidades de aprendizaje (ítem 8 $M=2,16$ vs 1,5$)$.

Las percepciones del GE en los siete ítems restantes fueron menos favorables al buen funcionamiento de las sesiones de tutoría, aunque sin significación estadística. Cuatro de estos ítems (9-12) se refieren a la contribución del tutor en el manejo del grupo y su aprendizaje, y los tres restantes (13-15) indican estrategias cognitivas y sociales empleadas en el grupo.

\section{Discusión}

En la UWS los problemas se construyen siguiendo los pasos del método clínico ${ }^{10}$, y se ejercitan habilidades de razonamiento necesarias para arribar a un correcto diagnóstico en el futuro ámbito laboral, en la búsqueda de soluciones al mismo. Este constante ciclo entre evocar lo conocido para iniciar el caso, detectar necesidades de aprendizaje, estudiar, y regresar al caso, coloca al problema, en cada sesión de tutoría, como inicio y fin del aprendizaje.

El agrado por las tutorías fue reportado en un mayor número de bloques por los estudiantes del GC que por los integrantes del GE (ítem 1); estos últimos con más frecuencia confesaron que los tópicos tratados tampoco les resultaron interesantes (ítem 2). En 5 estudiantes del GE y 3 del GC, el poco agrado por las tutorías coincidió con desinterés por los tópicos tratados en dos o más bloques; mientras que 11 estudiantes del GE (44\%) y 47 del GC (71\%) manifestaron interés por los tópicos y satisfacción por las tutorías, en 3 o en los 4 bloques. La selección de estas respuestas explora componentes que forman parte de las experiencias meta-cognitivas del estudiante en su grupo de tutoría ${ }^{11}$. El problema y la dinámica que su manejo desencadenó dentro del grupo generaron sentimientos de valencia positiva en más estudiantes del GC que en los del GE. Este interés, cuando es contagioso entre los integrantes del grupo, pudo mejorar la dedicación y compromiso en aquellos estudiantes que forman parte de esa "onda cognitiva-afectiva" 12 , la que no es estable y se ha comprobado que varía entre las diferentes fases por las que atraviesa la solución de un problema $^{13}$. Los porcentajes ya mostrados indican que ese sentimiento no fue exclusivo en los estudiantes del GC, también en el GE (44\%) hubo estudiantes que sintieron el efecto del agrado mezclado con el interés en sus tutorías.

La estrategia seguida por $64 \%$ de los estudiantes del GE en dos o más bloques fue la de seleccionar tópicos en lugar de estudiar todos los que su grupo detectó como necesidades de aprendizaje (ítem 3). Los individuos que se preparan mejor durante su estudio auto-regulado son capaces de detectar más "pistas" e insertarse con más frecuencia en las elaboraciones de su grupo y por lo tanto se benefician más en las mismas ${ }^{14}$. El no estudiar todos los objetivos de aprendizaje, también declarado por $26 \%$ de los integrantes del GC, si bien disminuye la carga cognitiva individual, también tiende a desplazar las interacciones verbales hacia modalidades de intercambio de menor demanda cognitiva, escenario que $\operatorname{Volet}^{8}$ describe como adquirir conocimiento en lugar de construir el significado de lo que se está aprendiendo. Los individuos con menos habilidades para planificar, ejecutar y modificar sus estrategias de aprendizaje necesitan más tiempo para procesar la información ${ }^{15}$, por lo que la selección de tópicos es un recurso que compensaría la falta de habilidad.

La encuesta no midió la participación en las interacciones, pero asumimos que aquellos que declararon sentir más inhibiciones al tiempo que percibieron menos oportunidades para hacerlo (ítem 4), efectivamente participaron con menor frecuencia. Muchos de los estudiantes de la UWS utilizan el idioma que es su medio de instrucción [L2 (inglés)] sólo cuando están en presencia de los docentes. Esto resulta en que es la lengua materna (L1) la que más se ejercita. Este alternante empleo de los dos idiomas afecta a los estudiantes con menos fluidez en L2 a participar en las interac- 
ciones verbales, en los que además repercute su velocidad de lectura y comprensión de los textos ${ }^{16}$, $\mathrm{y}$, consecuentemente, su rendimiento académico ${ }^{17}$.

Si un estudiante inhibido tiene poca fluidez en el inglés, ambas condiciones contribuyen a que con poca frecuencia se arriesgue a exponer sus puntos de vista en la tutoría. Individuos que no se sienten "a salvo" de cometer errores dentro del grupo, se auto-censuran y no expresan sus ideas por evitar el bochorno ${ }^{18}$.

Los argumentos que con más frecuencia exponen aquellos estudiantes que no han sido espontáneos al participar en la tutoría son: "porque no tenía (o lo creyó) información relevante para aportar", "otros estudiantes ya lo dijeron todo", "en cada sesión me propongo participar más pero no lo logro", "estudié los tópicos pero me cuesta trabajo explicarlos", razones que convergen con las reportadas por Kindler ${ }^{19}$; y contrastando con ellos están los estudiantes desinhibidos, los que con frecuencia emplean la estrategia de la re-vocalización ${ }^{20} \mathrm{o}$ se ofrecen a representar en diagramas o dibujos en la pizarra lo que otro estudiante verbalmente expone, y con estas estrategias ellos mismos se crean oportunidades para interactuar y aprender (ítem 5). Los estudiantes de la UWS, al igual que en otras universidades, necesitan que su tutor los ayude a manejar el desbalance que pueden crear los estudiantes dominantes dentro del grupo ${ }^{21}$. Esta ayuda de su tutor (ítem 6) fue percibida por más estudiantes del GC que por los del GE. El tutor debe ayudar a que habilidades ejercitadas por alumnos más capaces sean comprendidas ("traducidas") por el resto del grupo. Un ejemplo es re-visitar (prospectivo o retrospectivo) rutas de razonamiento a través de las cuales se arribó a una conclusión y, de este modo, descomponer los pasos críticos que llevan a la conclusión acertada. En ciertas fases de la solución del problema (ej. cuando se les entregan los resultados del examen físico, o de las investigaciones) el grupo puede subdividirse y analizar en tríos las tareas que serán discutidas algo más tarde en el grupo. El "trabajar acompañado" activa la interdependencia y cohesión entre los estudiantes, además de balancear el efecto que los grupos grandes tienen sobre el número de oportunidades que cada individuo tiene de participar ${ }^{22}$.

La autoevaluación de la comprensión del caso semanal (ítem 7) reveló que 56\% de los estudiantes del GE (vs 23\% GC) lo comprendió hasta en
2 bloques mientras que $44 \%$ (vs $77 \%$ GC) en tres o todos los bloques. La calidad del conocimiento que se construye y reconstruye en cada una de las sesiones por las que transita la solución de un problema tiene interdependencia cognitiva, de este modo, las fases precedentes afectan la calidad de las subsecuentes ${ }^{23}$. Escuchar las soluciones no tiene el mismo impacto cognitivo que activamente encontrarlas, lo que contribuiría a explicar el menor número de problemas comprendidos por los estudiantes del GE respecto a los del GC. Esa interdependencia cognitiva entre problemas, también permitiría que pueda ser comprendido en posteriores semanas, especulación que será objeto de un próximo estudio. La comprensión del problema tiene implícita múltiples transferencias desde las asignaturas que en él se integran de modo transdisciplinar, por lo que es un ejercicio cognitivo complejo, que si bien se logra a nivel grupal en la semana en la que transcurre, no lo es a nivel individual y los resultados encontrados lo demuestran.

En la UWS tutores y estudiantes priorizan la dimensión cognitiva de las tutorías ${ }^{24,25}$, selección que tiene soporte en la construcción de conocimiento que ocurre en el grupo facilitada por el tutor (ítems 11 y 12), pero es la dimensión social de la tutoría la que monitorea que algún individuo "no sea visible" dentro del grupo y su progreso.

La primera limitación del presente trabajo es que su análisis se sustentó en las percepciones de los individuos. Sin embargo, la subjetividad de las respuestas puede compensarse con la veracidad que se espera proviene del anonimato de la encuesta, a lo que se añade que en el momento de seleccionar la respuesta a cada ítem, el individuo se comparó consigo mismo en el transcurrir de los cuatro bloques de aprendizaje, lo que le permitió contrastar la percepción de su mejor bloque respecto al resto. Su segunda limitación es que el número de factores en el que se organizaron los ítems está avalado por valores del alfa ordinal en el rango de regulares, y la mayor fiabilidad se obtenía si se extraían sólo dos factores. La extracción de dos factores eliminaba algunos reactivos del análisis que, por ser importantes para los estudiantes, pueden contribuir al mejoramiento de las tutorías en ese contexto particular. Este criterio sustentó la no selección del modelo bi-factorial.

Los integrantes del GE tuvieron diferentes percepciones sobre el funcionamiento de las tutorías, 
en las que ellos compartieron con estudiantes del GC, y la dinámica que en sus grupos se desplegó no les resultó favorable para involucrarse en las interacciones verbales.

Las diferencias entre las medias de ambos grupos $(\mathrm{p}<0,05)$ se refieren a variables que midieron motivación y cognición individual, en un contexto donde las acciones de otros individuos, tutor incluido, fueron percibidas por los integrantes del GE, con mayor frecuencia, como no facilitadoras para la construcción de conocimiento individual.

Las tendencias anteriormente expresadas no deben opacar las diferencias entre los individuos (desviaciones estándares de las medias), las que alertan sobre la necesidad de desplegar estrategias diferentes (motivacionales, cognitivas, sociales) que complementen la necesidad individual.

\section{Referencias}

1. Iputo J. Tracking the Walter Sisulu University (South Africa) medical graduates-where are they 5 years after graduation? En: Assessing Health Professional Education (workshop summary). Washington: The National Academies Press; 2015. P.105. Disponible en: www. nap.edu/openblook.php? record_id $=18738$ \&page $=105$ [Consultado el 19 de febrero de 2015].

2. Walter Sisulu University; Faculty of Health Sciences. Prospectus 2014. Mthatha (South Africa): Walter Sisulu University; 2014.

3. Norman G, Dore K, Krebs J, Neville A. The power of the plural: effect of conceptual analogies on successful transfer. Academic Medicine 2007; 82 Suppl 10: S16-S18.

4. Schmidt H, Rotgans J, Yew E. The process of problem-based learning: what works and why. Medical Education 2011; 45 (8): 792-806.

5. Visschers-Pleijers A, Dolmans D, de Grave W, Wolfhagen I, Jacobs J, van der Vleuten C. Students perceptions about the characteristics of an effective discussion during the reporting phase in problem-based learning. Medical Education 2006; 40 (9): 924-31.

6. Keyton J, Stephenson J. Team attributes, processes, and values: a pedagogical framework. Business Communication Quarterly 2008; 71 (4): 488-504. Disponible en: http://web.ebscohost.com/ehost/pdfviewersid=82fed5b6-0138-4383-b34a-50b3662cff8e.35344544.pdf [Consultado el 6 de septiembre de 2013].

7. Frambach J, Driessen E, Beh P, van der Vleuten C. Quiet or questioning? Students' discussion behaviors in student-centered education across cultures. Studies in
Higher Education 2014; 39 (6): 1001-21.

8. Volet S, Summers M, Thurman J. High-level co-regulation in collaborative learning: How does it emerge and how is it sustained? Learning and Instruction 2009; 19 (2): 128-43.

9. Gari M, Iputo J. Opinión estudiantil sobre factores que influyen en el funcionamiento de las tutorías en la Universidad Walter Sisulu. Medicc Review 2015 (en prensa).

10. Ilizástigui F. El método clínico: muerte y resurrección. Revista Cubana de Educación Médica Superior 2000; 14 (2): 109-27. Disponible en: http://scieloprueba.sld. $\mathrm{cu} /$ scielo.php?.script=sci.pdf\&pid=S0864 [Consultado el 22 de enero de 2015].

11. Efklides A. Metacognition and affect: what can metacognitive experiences tell us about the learning process? Educational Research Review 2006; 1 (1): 3-14.

12. Linnenbrick-García L, Kempler TM, Koskey KL. Affect and engagement during small group instruction. Contemporary Educational Psychology 2011; 36 (1): 13-24.

13. Rotgan J, Schmidt H. Cognitive engagement in the problem-based learning classroom. Advances in Health Sciences Education 2011; 16 (4): 465-79.

14. Van Blankestein F, Dolmans D, van der Vleuten C, Schmidt H. Relevant prior knowledge moderates the effect of elaboration during small group discussion on academic achievement. Instructional Science 2013; 41 (4): 729-44.

15. Loyens S, Rikers R, Schmidt H. Relationships between students' conceptions of constructivist learning and their regulation and processing strategies. Instructional Science 2008; 36 (5-6): 445-62.

16. Nergis A. Exploring the factors that affect reading comprehension of EAP learners. Journal of English for Academic Purposes 2013; 12: 1-9. Disponible en: http://ac.els.cdn.com/S1475158512000550/1-r2.0S1475158512000550-main.pdf [Consultado el 12 de diciembre de 2013].

17. Singaram V, van der Vleuten C, Muijtjens A, Dolmans D. Relationship between language background, secondary school scores, tutorial group processes, and students' academic achievement in PBL: testing a causal model. Interdisciplinary Journal of Problem-based Learning 2012; 6 (1): 153-64. Disponible en: http://dx.doi. org/10.771/1541-5015.1316 [Consultado el 5 de enero de 2014].

18. Edmondton A. Psychological safety and learning behavior in work teams. Administrative Science Quarterly 1999; 44 (2): 350-83.

19. Kindler P, Grant Ch, Kulla S, Poole G, Godolphin W. Difficult incidents and tutor interventions in problem 
based learning. Medical Education 2009; 43(9): 866-73.

20. O’Connor M, Michaels S. Aligning academic task and participation status through revoicing: Analysis of a classroom discourse strategy. Anthropology and Education Quarterly 1993; 24 (4): 318-35.

21. Hendry G, Ryan G, Harris J. Group problems in problem-based learning. Medical Teacher 2003; 25 (6): 609-16.

22. Ruey-Shiang S. The relationships between group size, participation, and performance of programming language learning supported with online forums. Computers and Education 2013; 62: 196-207. Disponible en: http:// dx.doi.org/10.1016/j.compedu.2012.11.001 [Consultado el 26 de enero de 2015].
23. Yew E, Chng E, Schmidt H. Is learning in problem-based learning cumulative? Advances in Health Sciences Education 2011; 16 (4): 449-69.

24. Iputo J. Facilitating the integrated small group tutorial in a medical programme-the University of Transkei (Unitra) experience. South African Medical Journal 2005; 95 (12): 959-62.

25. Gari M, Rivera N. Las acciones del tutor en el aprendizaje basado en la solución de problemas en una Universidad rural de África del Sur. Revista de Docencia Universitaria. REDU. Número monográfico dedicado a tutorías y sistemas de apoyo a los estudiantes 2013; 11 (2): 153-71. Disponible en: http://red-u.net [Consultado el 25 de noviembre de 2014 . 